\title{
Fermi Energy-Incorporated Generalized BCS Equations for the Temperature-Dependent Critical Current Density and the Related Parameters of a Superconductor for All $T \leq T_{c}$ and Their Application to Aluminium Strips
}

\author{
Gulshan Prakash Malik ${ }^{1 *}$, Vijaya Shankar Varma ${ }^{2 \#}$ \\ ${ }^{1}$ B-208 Sushant Lok 1, Gurgaon, Haryana, India \\ ${ }^{2} 180$ Mall Apartments, Mall Road, Delhi, India \\ Email: gulshanpmalik@yahoo.com,varma2@gmail.com
}

\begin{abstract}
How to cite this paper: Malik, G.P. and Varma, V.S. (2019) Fermi Energy-Incorporated Generalized BCS Equations for the Temperature-Dependent Critical Current Density and the Related Parameters of a Superconductor for All $T \leq T_{c}$ and Their Application to Aluminium Strips. World Journal of Condensed Matter Physics, 9. 47-61.
\end{abstract}

https://doi.org/10.4236/wjcmp.2019.93004

Received: June 25, 2019

Accepted: July 20, 2019

Published: July 23, 2019

Copyright $\odot 2019$ by author(s) and Scientific Research Publishing Inc. This work is licensed under the Creative Commons Attribution International License (CC BY 4.0).

http://creativecommons.org/licenses/by/4.0/

(c) (i) Open Access

\begin{abstract}
Presented here are the Generalized BCS Equations incorporating Fermi Energy for the study of the $\left\{\Delta, T_{\mathcal{o}} j_{c}(T)\right\}$ values of both elemental and composite superconductors (SCs) for all $T \leq T_{c}$ where $\Delta, T_{c}$ and $j_{c}(T)$ denote, respectively, one of the gap values, the critical temperature and the $T$-dependent critical current density. This framework, which extends our earlier study that dealt with the $\left\{\Delta_{0}, T_{c}, j_{c}(0)\right\}$ values of an SC, is also shown to lead to $T$-dependent values of several other related parameters such as the effective mass of electrons, their number density, critical velocity, Fermi velocity $\left(V_{F}\right)$, coherence length and the London penetration depth. The extended framework is applied to the $j_{c}(T)$ data reported by Romijn et al. for superconducting Aluminium strips and is shown not only to provide an alternative to the explanation given by them, but also to some novel features such as the role of the Sommerfeld coefficient $\gamma(T)$ in the context of $j_{c}(T)$ and the role of $V_{F}(T)$ in the context of a recent finding by Plumb et al. about the superconductivity of $\mathrm{Bi}-2212$.
\end{abstract}

\section{Keywords}

$j_{c}(T)$ Data for Superconducting Aluminium Strips, Bardeen's Equation and Kupriyanov-Lukichev Theory for $j_{c}(T)$, Unified Generalized BCS Equations for $\left\{\Delta, T_{\mathcal{c}} j_{c}(T)\right\}, T$-Dependence of the Sommerfeld Coefficient and Several Other Superconducting Parameters

\footnotetext{
${ }^{\star}$ Formerly of the School of Environmental Sciences, Jawaharlal Nehru University, New Delhi, India.
}

"Formerly of the Department of Physics and Astrophysics, University of Delhi, Delhi, India. 


\section{Introduction}

Adopting the framework of the Fermi energy $\left(E_{F}\right)$-incorporated generalized BCS equations (GBCSEs), we deal here with the calculation of the critical current density $j_{c}(T)$-for all $\mathrm{T}$ between 0 and $T_{c}$-of a superconductor (SC) which is not subjected to any external magnetic field. Specifically, we address here the data obtained by Romijn et al. [1] for superconducting aluminium strips for which it suffices to apply GBCSEs in the scenario where Cooper pairs (CPs) are formed via the one-phonon exchange mechanism (1 PEM). However, with high- $T_{c}$ SCs in mind, also given here are GBCSEs that enable one to deal in a unified manner with the gaps $(\Delta \mathrm{s}), T_{c}$ and $j_{c}(T)$ of a composite, multi-gapped SC requiring more than 1 PEM.

The paper is organized as follows. In order to provide a perspective of the conceptual basis of the conventional, multi-band approach (MBA) to the study of the set $\left\{\Delta, T_{\mathcal{o}} j_{c}(T)\right\}$ of a composite SC vis-à-vis that of the GBCSEs-based approach, we include in this section an overview of both approaches. Since the data in [1] are explicable in the conventional approach via both the phenomenological Bardeen equation [2] and the Kupriyanov and Lukichev (KL) [3] theory discussed below, the purpose of this paper is to show that the GBCSEs-based approach provides a valuable alternative explanation of the same data. In the next section are given the $E_{F}$-incorporated GBCSEs in the scenario when a two-phonon exchange mechanism (2 PEM) is operative. These equations provide a unified framework for the description of the set $\left\{\Delta_{2}, T_{\mathcal{c}} j_{0}\right\}$, where $\Delta_{2}$ is the larger of the two gaps of the SC. Application of the GBCSEs to the $j_{c}(T)$ data in [1] is taken up in Section 3 where they are also shown to provide the values of several other related $T$-dependent parameters such as the Sommerfeld coefficient, the effective mass of electrons, their number density, critical velocity, Fermi velocity, coherence length and the London penetration depth. Sections 4 and 5 are devoted, respectively, to a Discussion of our approach and the Conclusions following from it.

\subsection{Overview of the Conventional Approach in Dealing with the Set $\left\{\Delta, T_{c}, j_{c}(T)\right\}$ of a Composite SC}

The gaps of a hetero-structured SCs are most widely studied via an adaptation of the multi-band approach (MBA) of Suhl et al. [4]. When such a study also deals with a $T_{c}$ of the SC, which is not always the case, it generally appeals to the Migdal-Eliashberg-McMillan approach (MEMA) [5]. Even though MEMA is cast in the BCS mould of a 1-phonon exchange mechanism (1 PEM) for the formation of CPs, it is employed to deal with such high- $T_{c} s$ as are observed because it permits the BCS interaction parameter $\lambda$ to even exceed unity because it is based on an integral equation the expansion parameter of which is not $\lambda$, but $m_{e} / M$ where $m_{e}$ is the electron mass and $M$ the mass of an ion. Insofar as $j_{c}$ is concerned, a plethora of formulae is conventionally employed depending upon the type of the SC (I or II), its size, shape and the manner of preparation-for some such formulae which do not take into account the $T$-dependence of $j_{c}$ see [6]. 
Insofar as $j_{c}(T)$ is concerned, it is empirically known that it has its maximum value at $T=0$ and that $j_{c}\left(T_{c}\right)=0$. It took considerable time for theoretical attempts to evolve before the observed variation of $j_{c}(T)$ between these limits could be explained. Perhaps the earliest such attempt was due to London, who gave an equation for $j_{c}(T)$ valid at $T=T_{c}$ but which failed at lower temperatures because, as was later realized, it did not take into account the effect of the change in the order parameter with current/temperature. The equation for $j_{c}(T)$ given by the phenomenological Ginzberg-Landau (GL) theory marks the next stage in the said evolution. This equation works well close to $T_{\mathcal{c}}$, but not for much lower temperatures. As is well known, the GL theory reduces to the London theory when the concentration of superconducting electrons is uniformly distributed and that, as was shown by Gor'kov, the microscopic theory in which the energy gap is taken as an order parameter leads to the GL theory near $T_{c}$. These brief considerations suggest the need to appeal to the microscopic theory in order to explain the observed variation of $j_{c}(T)$ for all $T \leq T_{c}$ Before we do so, it is relevant to draw attention to a phenomenological equation for $j_{c}(T)$ given by Bardeen [2] post-BCS. This equation, valid for all $T$ between 0 and $T_{c}$ and obtained by treating the gap as a variational parameter and minimizing the free energy for a given current, is

$$
j_{c}(T)=\frac{1}{2 \sqrt{2}} j_{c}(0)\left[1-\left(T / T_{c}\right)^{2}\right]^{3 / 2} .
$$

Equation (1) is applicable to SCs for which changes in the energy gap with position can be neglected.

Since the thin samples of Romijn et al. [1] satisfied the condition(s) of validity of (1), they applied it to one of their samples and found that it indeed fits their data well. Nonetheless, (1) does not address the core issue of the problem, i.e., to identify the parameters on which $j_{c}(T)$ depends. The knowledge of these is essential because it provides a handle to control $j_{c}(T)$. To unravel what lies beneath the "blanket" of (1), one needs a microscopic theory, viz. the theory given by Eilenberger which is derived from the original Gor'kov theory under the assumption $\rho_{F} \ell \gg 1$, where $\rho_{F}$ is the electrical resistivity of electrons at the Fermi surface and $\ell$ their mean free path. It was shown by KL [3] that for an SC subject to certain constraints, the Eilenberger equations can be further simplified. Since their samples satisfied these conditions, Romijn et al. [1] also employed approximate solutions of the KL equations and found that their data were thus adequately explained.

\subsection{Overview of the GBCSEs-Based Approach in Dealing with the Set $\left\{\Delta, T_{c}, j_{c}(T)\right\}$ of a Composite SC}

Complementing MBA and presented in a recent monograph [7] is an approach based on the GBCSEs which too has been applied to a significant number of SCs. One of the premises of this approach is that Fermi energy $\left(E_{F}\right)$ plays a fundamental role in determining the superconducting properties of an SC. We recall 
in this connection that the usual BCS equations for the $T_{c}$ and $\Delta$ of an elemental SC are independent of $E_{F}$ because of the assumption that $E_{F} \gg k \theta$, where $k$ is the Boltzmann constant and $\theta$ the Debye temperature of the SC. GBCSEs are obtained via the Matsubara technique and a Bethe-Salpeter equation (BSE) the kernel of which is a super propagator. The latter feature leads to the characterization of a composite SC by CPs with multiple binding energies $(|W| s)$. A salient feature of this approach is that it invariably invokes a $\lambda$ for each of the ion-species that may cause pairing, whence one has the same $\lambda s$ in the equations for any $\Delta$ and the corresponding $T_{c}$ of the SC-as is the case for elemental SCs. Multiple gaps arise in this approach because different combinations of $\lambda$ s operate on different parts of the Fermi surface due to its undulations. Each of the $|W|$ s so obtained is identified with a $\Delta$ of the SC. Thus, as shown in [7], with the input of the values of any two gaps of an SC and a value of its $T_{\mathcal{o}}$ this approach goes on to shed light on several other values of these parameters. This is not so for MBA, another feature of which is that even when it is employed to deal with the same SC by different authors, the number of bands invoked is not always the same.

\section{2. $E_{F}$-Incorporated GBCSEs for the $T_{c}, \Delta s$ and $j_{c}$ of a Hetero-Structured SC}

\subsection{Equations for $\left|W_{20}\right|$ (To Be Identified with $\Delta_{20}$, the Larger of the Two Gaps at $T=0), W_{2}\left(t=T / T_{c}\right)$ and $T_{c}$ [7]}

The equation for $\left|W_{20}\right|$ is:

$$
\frac{\lambda_{1}}{2} a_{1}\left(\theta_{1}, \mu, W_{20}\right)+\frac{\lambda_{2}}{2} a_{2}\left(\theta_{2}, \mu, W_{20}\right)=\left[\frac{3}{4} a_{3}\left(\mu, W_{20}\right)\right]^{1 / 3},
$$

where

$$
\begin{aligned}
& a_{1}\left(\theta_{1}, \mu, W_{20}\right)=\boldsymbol{R} \boldsymbol{e}\left[\int_{-k \theta_{1}}^{k \theta_{1}} \mathrm{~d} \xi \frac{\sqrt{\xi+\mu}}{|\xi|+\left|W_{20}\right| / 2}\right], a_{2}\left(\theta_{2}, \mu, W_{20}\right)=a_{1}\left(\theta_{2}, \mu, W_{20}\right) \\
& a_{3}\left(\theta_{m}, \mu, W_{20}\right)=\boldsymbol{R e}\left[\frac{4}{3}\left(\mu-k \theta_{m}\right)^{3 / 2}+\int_{-k \theta_{m}}^{k \theta_{m}} \mathrm{~d} \xi \sqrt{\xi+\mu}\left(1-\frac{\xi}{\sqrt{\xi^{2}+W_{20}^{2}}}\right)\right] .
\end{aligned}
$$

The equation for $\left|W_{2}(t)\right|$ for $0<t<1$ is:

$$
\lambda_{1} b_{1}\left(\theta_{1}, \mu, W_{2}\right)+\lambda_{2} b_{2}\left(\theta_{2}, \mu, W_{2}\right)=\left[\frac{3}{4} b_{3}\left(\theta_{m}, \mu, W_{2}\right)\right]^{1 / 3},
$$

where

$$
\begin{gathered}
b_{1}\left(\theta_{1}, \mu, W_{2}\right)=b_{11}\left(\theta_{1}, \mu, W_{2}\right)+b_{12}\left(\theta_{1}, \mu, W_{2}\right) \\
b_{2}\left(\theta_{2}, \mu, W_{2}\right)=b_{21}\left(\theta_{2}, \mu, W_{2}\right)+b_{22}\left(\theta_{2}, \mu, W_{2}\right) \\
b_{11}\left(\theta_{1}, \mu, W_{2}\right)=\int_{-k \theta_{1}}^{0} \mathrm{~d} \xi \sqrt{\mu+\xi} \frac{\tanh \left[\left(1 / 2 k t T_{c}\right)\left(\xi-W_{2}\right)\right]}{\xi-W_{2}} \\
b_{12}\left(\theta_{1}, \mu, W_{2}\right)=\int_{0}^{k \theta_{1}} \mathrm{~d} \xi \sqrt{\mu+\xi} \frac{\tanh \left[\left(1 / 2 k t T_{c}\right)\left(\xi+W_{2}\right)\right]}{\xi+W_{2}}
\end{gathered}
$$




$$
\begin{aligned}
& b_{21}\left(\theta_{2}, \mu, W_{2}\right), b_{22}\left(\theta_{2}, \mu, W_{2}\right)=b_{11}\left(\theta_{2}, \mu, W_{2}\right), b_{12}\left(\theta_{2}, \mu, W_{2}\right) \\
& b_{3}\left(\theta_{m}, \mu, W_{2}\right)=\int_{-\mu}^{k \theta_{m}} \sqrt{\mu+\xi}\left[1-\frac{\xi \tanh \left[\left(1 / 2 k t T_{c}\right) \sqrt{\xi^{2}+W_{2}^{2}}\right]}{\sqrt{\xi^{2}+W_{2}^{2}}}\right],
\end{aligned}
$$

and the equation for $T_{c}$ is:

$$
\frac{\lambda_{1}}{2} c_{1}\left(\theta_{1}, \mu, T_{c}\right)+\frac{\lambda_{2}}{2} c_{2}\left(\theta_{2}, \mu, T_{c}\right)=\left[\frac{3}{4} c_{3}\left(\theta_{m}, \mu, T_{c}\right)\right]^{1 / 3},
$$

where

$$
\begin{aligned}
& c_{1}\left(\theta_{1}, \mu, T_{c}\right)=\boldsymbol{R e}\left[\int_{-\theta_{1} / 2 T_{c}}^{\theta_{1} / 2 T_{c}} \mathrm{~d} x \sqrt{2 k T_{c} x+\mu} \frac{\tanh (x)}{x}\right] \\
& c_{2}\left(\theta_{2}, \mu, T_{c}\right)=c_{1}\left(\theta_{2}, \mu, T_{c}\right) \\
& c_{3}\left(\theta_{m}, \mu, T_{c}\right)=\boldsymbol{R e}\left[2 k T_{c} \int_{-\theta_{m} / 2 T_{c}}^{\theta_{m} / 2 T_{c}} \mathrm{~d} x \sqrt{2 k T_{c} x+\mu}\{1-\tanh (x)\}\right]
\end{aligned}
$$

In the above equations, $\theta_{1}$ and $\theta_{2}$ are the Debye temperatures of the ion-species that cause pairing and are obtained from the Debye temperature $\theta$ of the SC as detailed in [7] and [8]; $\theta_{m}$ is the greater of the temperatures $\theta_{1}$ and $\theta_{2}$, and the variation of chemical potential with temperature has been ignored in order to avoid the situation where we have an under-determined set of equations. Thus, we have assumed that the chemical potential $\mu(T=0)=\mu\left(T=T_{c}\right)=E_{F}$. With the input of $\left|W_{20}\right|, T_{\mathcal{o}}$ and different assumed values of $\mu=\rho k \theta \quad(\rho=100,50,25$, 10,5 , etc.), solution of simultaneous Equations (2) and (4) yields, for each value of $\mu$, the corresponding values of the interaction parameters $\lambda_{1}$ and $\lambda_{2}$.

\subsection{Equation for a Dimensionless Construct $y_{0}$ at $T=0$ Which Enables One to Calculate $j_{0}$ and Several Other Superconducting Parameters}

The construct $y_{0}$ is defined as

$$
y_{0}=\left(k \theta / P_{0}\right) \sqrt{2 m^{*}(0) / E_{F}},
$$

where $m^{\star}(0)$ is the effective mass of superconducting electrons at $T=0$ and $P_{0}$ their critical momentum.

The exercise carried out above leads to a multitude of values for the set $S=\left\{E_{F}\right.$, $\left.\lambda_{1}, \lambda_{2}\right\}$, each of which is consistent with the $\left|W_{20}\right|$ and $T_{c}$ values of the SC. In order to find the unique set of values from among them that also leads to the empirical value of $j_{0}$ of the SC, we solve the following equation for $y_{0}$ [9] for each triplet of $\left\{E_{F}, \lambda_{1}, \lambda_{2}\right\}$ values:

$$
1=\frac{\lambda_{1}}{4} J\left(\Sigma_{1}^{\prime}, r_{1} y_{0}\right)+\frac{\lambda_{2}}{4} J\left(\Sigma_{2}^{\prime}, r_{2} y_{0}\right),
$$

where

$$
\begin{aligned}
& J\left(\Sigma^{\prime}, y_{0}\right)=\boldsymbol{R} \boldsymbol{e}\left[\int_{0}^{1} \mathrm{~d} x F\left(\Sigma^{\prime}, x, y_{0}\right)\right], F\left(\Sigma^{\prime}, x, y_{0}\right)=f_{1}\left(\Sigma^{\prime}, x, y_{0}\right)+f_{2}\left(\Sigma^{\prime}, x, y_{0}\right) \\
& f_{1}\left(\Sigma^{\prime}, x, y_{0}\right)=4\left[-u_{1}\left(\Sigma^{\prime}, x, y_{0}\right)-u_{2}\left(\Sigma^{\prime}, x, y_{0}\right)+u_{3}\left(\Sigma^{\prime}, x, y_{0}\right)+u_{4}\left(\Sigma^{\prime}, x, y_{0}\right)\right]
\end{aligned}
$$




$$
\begin{aligned}
& f_{2}\left(\Sigma^{\prime}, x, y_{0}\right) \\
& =2 \ln \left[\frac{1+u_{1}\left(\Sigma^{\prime}, x, y_{0}\right)}{1-u_{1}\left(\Sigma^{\prime}, x, y_{0}\right)} \frac{1+u_{2}\left(\Sigma^{\prime}, x, y_{0}\right)}{1-u_{1}\left(\Sigma^{\prime}, x, y_{0}\right)} \frac{1-u_{3}\left(\Sigma^{\prime}, x, y_{0}\right)}{1+u_{3}\left(\Sigma^{\prime}, x, y_{0}\right)} \frac{1-u_{4}\left(\Sigma^{\prime}, x, y_{0}\right)}{1+u_{4}\left(\Sigma^{\prime}, x, y_{0}\right)}\right] \\
& u_{1}\left(\Sigma^{\prime}, x, y_{0}\right)=\sqrt{1-\Sigma^{\prime} x / y_{0}}, u_{2}\left(\Sigma^{\prime}, x, y_{0}\right)=\sqrt{1+\Sigma^{\prime} x / y_{0}} \\
& u_{3}\left(\Sigma^{\prime}, x, y_{0}\right)=\sqrt{1-\Sigma^{\prime}\left(1-x / y_{0}\right)}, u_{4}\left(\Sigma^{\prime}, x, y_{0}\right)=\sqrt{1+\Sigma^{\prime}\left(1-x / y_{0}\right)} \\
& \Sigma^{\prime}=k \theta / E_{F}, \Sigma_{i}^{\prime}=k \theta_{i} / E_{F}, r_{i}=\theta_{i} / \theta
\end{aligned}
$$

The operator $\boldsymbol{R} \boldsymbol{e}$ ensures that the integrals yield real values even when expressions under the radical signs are negative (as happens for the heavy fermion SCs).

Corresponding to each value of $y_{0}$ obtained by solving (6) with the input of $\{\theta$, $\left.\theta_{1}, \theta_{2}, E_{F}, \lambda_{1}, \lambda_{2}\right\}$, we can calculate several superconducting parameters in terms of $\theta, E_{P}, y_{0}$, the gram-atomic volume $V_{g}$ and the electronic heat constant/Sommerfeld coefficient $\gamma_{0}$ at $T=0$ by employing the following relations, see [8] and for a correction, [10]:

$$
\begin{gathered}
s(0) \equiv m^{*}(0) / m_{e}=A_{1} \frac{\left(\gamma_{0} / v_{g}\right)^{2 / 3}}{E_{F}^{1 / 3}} \\
N_{s 0}\left(E_{F}\right)=A_{2}\left(\gamma_{0} / v_{g}\right) E_{F} \\
P_{0}\left(E_{F}\right)=A_{3} \frac{\theta}{y_{0}} \frac{\left(\gamma_{0} / v_{g}\right)^{1 / 3}}{E_{F}^{2 / 3}} \\
V_{c 0}\left(E_{F}\right)=A_{4} \frac{\theta}{y_{0}} \frac{1}{\left(\gamma_{0} / v_{g}\right)^{1 / 3} E_{F}^{1 / 3}} \\
j_{0}\left(E_{F}\right)=\frac{N_{s 0}\left(E_{F}\right)}{2} e^{*} V_{c 0}\left(E_{F}\right)=A_{5} \frac{\theta}{y_{0}}\left(\gamma_{0} / v_{g}\right)^{2 / 3} E_{F}^{2 / 3}, \quad\left(e^{*}=2 e\right)
\end{gathered}
$$

where $m_{e}$ is the free electron mass and $e$ the electronic charge,

$$
\begin{gathered}
A_{1} \cong 3.305 \times 10^{-10} \mathrm{eV}^{-1 / 3} \cdot \mathrm{cm}^{2} \cdot \mathrm{K}^{4 / 3}, A_{2} \cong 2.729 \times 10^{7} \mathrm{eV}^{-2} \cdot \mathrm{K}^{2}, \\
A_{3} \cong 1.584 \times 10^{-6} \mathrm{~cm} \cdot \mathrm{K}^{-1 / 3}, A_{4} \cong 1.406 \times 10^{8} \mathrm{eV}^{2 / 3} \cdot \mathrm{sec}^{-1} \cdot \mathrm{K}^{-5 / 3}, \\
A_{5} \cong 6.146 \times 10^{-4} \mathrm{C} \cdot \mathrm{eV}^{-4 / 3} \cdot \mathrm{K}^{1 / 3} \cdot \mathrm{s}^{-1},
\end{gathered}
$$

and $N_{s 0}$ is the number density of CPs and $V_{c 0}$ their critical velocity at $T=0$. Comparison of the $j_{0}$-values so obtained with the experimental value of $j_{0}$ then leads to the desired unique set of $\left\{E_{F}, \lambda_{1}, \lambda_{2}\right\}$-values that is consistent with the empirical values of $\left|W_{20}\right|, T_{\mathcal{c}}$ and $j_{0}$ of the SC.

\subsection{Equation for the Dimensionless Construct}

$$
\begin{aligned}
& y(t)=\left(k \theta / P_{c}(t)\right) \sqrt{2 m^{*} / E_{F}(t)} \text { Which Enables One } \\
& \text { to Calculate } j_{c}(t) \text { and Several Other Superconducting } \\
& \text { Parameters at } t \neq 0
\end{aligned}
$$

When $t \neq 0$, the equation for $y(t)$ is obtained from [9] 


$$
\begin{aligned}
1= & \frac{\lambda_{1}}{4} \int_{0}^{1} \mathrm{~d} x\left[I_{1}\left(x, \theta_{1}, E_{2}\right)+I_{2}\left(x, \theta_{1}, E_{2}\right)\right] \\
& +\frac{\lambda_{2}}{4} \int_{0}^{1} \mathrm{~d} x\left[I_{1}\left(x, \theta_{2}, E_{2}\right)+I_{2}\left(x, \theta_{2}, E_{2}\right)\right],
\end{aligned}
$$

where

$$
\begin{aligned}
& I_{1}\left(x, \theta, E_{2}\right)=\int_{-E_{1}+E_{2} x}^{E_{1}-E_{2} x} \mathrm{~d} \xi \phi(\xi) \tanh \left[(\beta / 2)\left(\xi+E_{2} x\right)\right] \\
& I_{2}\left(x, \theta, E_{2}\right)=\int_{-E_{1}+E_{2} x}^{E_{1}-E_{2} x} \mathrm{~d} \xi \phi(\xi) \tanh \left[(\beta / 2)\left(\xi-E_{2} x\right)\right] \\
& \phi(\xi)=\sqrt{1+\xi / E_{F}} /\left(\xi+E_{3}\right), E_{1}=k \theta \\
& E_{2}(t)=P_{c}(t) \sqrt{E_{F} / 2 m^{*}(t)}, E_{3}(t)=P_{c}^{2}(t) / 8 m^{*}(t), \beta=1 / k t T_{c} .
\end{aligned}
$$

In terms of $y(t)=E_{1} / E_{2}(t)=\left[k \theta / P_{c}(t)\right] \sqrt{2 m^{*}(t) / E_{F}}$, we have (12) as:

$$
1=\frac{\lambda_{1}}{4} \int_{0}^{1} \mathrm{~d} x f\left(x, \theta_{1}, E_{F}, r_{1} y\right)+\frac{\lambda_{2}}{4} \int_{0}^{1} \mathrm{~d} x f\left(x, \theta_{2}, E_{F}, r_{2} y\right),
$$

where

$$
\begin{aligned}
& f\left(x, \theta, E_{F}, y\right) \\
& =\int_{-E_{1}(1-x / y)}^{E_{1}(1-x / y)} \mathrm{d} \xi \phi\left(\xi, E_{F}, y\right)\left\{\tanh \left[\frac{\theta}{2 t T_{c} y}\left(\frac{\xi y}{k \theta}+x\right)\right]+\tanh \left[\frac{\theta}{2 t T_{c} y}\left(\frac{\xi y}{k \theta}-x\right)\right]\right\} \\
& E_{1}=k \theta, \phi\left(\xi, E_{F}, y\right)=\sqrt{1+\xi / E_{F}} /\left[\xi+E_{3}(y)\right], \\
& E_{3}(y)=E_{1}^{2} / 8 m^{*} \alpha^{2} y^{2}, r_{i}=\theta_{i} / \theta
\end{aligned}
$$

Each value of $y(t)$ obtained by solving (13) for different values of the set $S=$ $\left\{E_{F}, \lambda_{1}, \lambda_{2}\right\}$ leads to the corresponding value of $j_{c}(t)$ via (11), and to the values of the associated parameters via (7)-(10).

\section{Explanation of the Empirical $j_{c}(t)$ Values of Aluminium Strips Via GBCSEs}

By adding to the RHS of each of the Equations (2), (3), and (4) a term in which $\lambda_{2}$ and $\theta_{2}$ are replaced by $\lambda_{3}$ and $\theta_{3}$, respectively, the above framework is easily extended to a 3PEM scenario where pairing is caused by three ion-species. On the other hand, in order to deal with the data reported for Aluminium strips in [1], we need the reduced framework of 1 PEM. This is obtained by putting $\lambda_{2}=0$ in each of the three equations just noted.

\subsection{The $j_{0}$ Data}

Reported in [1] are various superconducting properties for six samples of aluminium strips at $T=0$. Among these, $T_{c}$ and $j_{0}$ are determined experimentally, whereas values of some other parameters such as coherence length $\xi$ and the London penetration depth $\lambda_{L}$ are model-dependent derived properties.

In order to show how the GBCSEs-based approach works in such a situation, we give below the sequential steps that are followed for Sample 1 in [1]. 
1) The $T_{c}$ of the sample is $1.196 \mathrm{~K}$. We take its Debye temperature $\theta$ to be 428 $\mathrm{K}$ [11]. Employing these values and putting $\lambda_{2}=0$ (as is appropriate for a 1 PEM), solution of (4) for some typical values of $E_{F}$ are: $\lambda_{1}=0.1665$ for any value of $E_{F}=10-100 \mathrm{k} \theta, \lambda_{1}=0.1666$ for $E_{F}=5 \mathrm{k} \theta, \lambda_{1}=0.1670$ for $E_{F}=2 \mathrm{k} \theta$.

2) For some select pairs of $\left\{E_{F}, \lambda_{1}\right\}$ values obtained above, we now solve (6) for the corresponding value of $y_{0}$ and obtain the following results (in the parentheses are given the $\left\{E_{F}, \lambda_{1}\right\}$ values): $y_{0}=149.8\{50 \mathrm{k} \theta, 0.1665\} ; y_{0}=149.9\{25 \mathrm{k} \theta$, $0.1665\} ; y_{0}=149.9\{10 \mathrm{k} \theta, 0.1665\} ; y_{0}=149.6\{5 \mathrm{k} \theta, 0.1666\} ; y_{0}=149.5\{2 \mathrm{k} \theta$, $0.1670\}$.

3) For each of the above pairs of $\left\{E_{F}, y_{0}\right\}$ values, we now calculate $j_{0}$ via (11). For this purpose, besides the value of $\theta$, we require the values of $\gamma_{0}$ and $v_{g}$ which are taken as: $\gamma_{0}=1.36 \mathrm{~mJ} / \mathrm{mol}-\mathrm{K}^{2}[11], V_{g}=10 \mathrm{~cm}^{3} /$ gram-atom. The results in $\mathrm{A} / \mathrm{cm}^{2}$ with the corresponding $\left\{E_{F}, y_{0}\right\}$ values given in parentheses are: $2.37 \times 10^{7}$ $\{50 \mathrm{k} \theta, 149.8\}, 1.49 \times 10^{7}\{25 \mathrm{k} \theta, 149.8\} 8.09 \times 10^{6}\{10 \mathrm{k} \theta, 149.9\}, 5.11 \times 10^{6}\{5 \mathrm{k} \theta$, $149.6\}, 2.77 \times 10^{6}\{2 \mathrm{k} \theta, 149.5\}$.

4) Among the above values, $j_{0}=1.49 \times 10^{7} \mathrm{~A} / \mathrm{cm}^{2}$ corresponding to $\left\{E_{F}=25 \mathrm{k} \theta\right.$, $\left.y_{0}=149.8\right\}$ is closest to the experimental value of $1.53 \times 10^{7} \mathrm{~A} / \mathrm{cm}^{2}$. By fine-tuning the value of $E_{F}$ and repeating the above exercise, we find that the experimental value of $j_{0}$ is obtained exactly when $E_{F}=26 \mathrm{k} \theta=0.959 \mathrm{eV}$ and $y_{0}=$ 149.8 .

5) With $E_{F}$ fixed at this value, we can find $W_{10}$ via (2) (with $\lambda_{2}=0$ ).

6) With $E_{F}$ and $y_{0}$ of the sample fixed as above, we can calculate the values of $s(0), N_{s}(0)$, and $V_{c 0}$ via (7), (8) and (10), respectively, and the Fermi velocity $V_{F 0}$ at $T=0$ via the relation

$$
(1 / 2) m^{*}(0) V_{F 0}^{2}=E_{F} \text {. }
$$

Besides these parameters, we can now also calculate the coherence length $\xi_{0}$ at $T=0$ via

$$
\xi_{0}=\hbar V_{F 0} / \pi\left|W_{10}\right|,
$$

and the London penetration depth $\lambda_{L 0}$ at $T=0$ via

$$
\lambda_{L 0}=\sqrt{m^{*}(0) c m^{3}(\hbar c)^{2} / 4 \pi n_{s}(0) e^{2}},
$$

where units for $m^{*}$ and $n_{s}$ are electron-volt and $\mathrm{cm}^{-3}$, respectively, $e=(137.03604)^{-1 / 2}$, $\mathrm{cm}=5.067728861 \times 10^{4}(\hbar c) \mathrm{eV}^{-1}$ and, for convenience of the reader, we have inserted the requisite factors of $\hbar$ and $c$ in order to obtain the value of $\lambda_{L 0}$ in cgs units. The results of these calculations are given in Table 1, which also gives similar results for the remaining five samples dealt with in [1].

\subsection{The $j_{c}(t)$ Data}

Insofar as $j_{c}(t)$ is concerned, Romijn et al. [1] have given results for one of their samples (Sample 5) in their Figure 4, which is a plot of their experimental values of $\left[j_{c}(t) / j_{c}(0)\right]^{2 / 3}$ along with their counterparts as obtained via the phenomenological Bardeen Equation (1) and the KL theory [3]. It follows from this 
Table 1. For the samples employed in [1], the $T=0$ values of various superconducting parameters obtained via the procedure detailed in Section 3.1.

\begin{tabular}{|c|c|c|c|c|c|c|c|c|c|c|c|c|c|}
\hline Sample & $\begin{array}{r}T_{c} \\
(\mathrm{~K})\end{array}$ & $\begin{array}{c}j_{0} \\
(\exp ) \\
\left(10^{7} \mathrm{~A} / \mathrm{cm}^{2}\right)\end{array}$ & $\begin{array}{l}j_{0} \text { (theory) } \\
\left(10^{7} \mathrm{~A} / \mathrm{cm}^{2}\right)\end{array}$ & $\begin{array}{c}\rho \\
\left(E_{F}=\rho k \theta\right)\end{array}$ & $\lambda_{1}$ & $y_{0}$ & $s$ & $\begin{array}{c}N_{s}(0) \\
\left(10^{22} \mathrm{~cm}^{-3}\right)\end{array}$ & $\begin{array}{c}V_{0} \\
\left(10^{3} \mathrm{cms}^{-1}\right)\end{array}$ & $\begin{array}{c}V_{F} \\
\left(10^{7} \mathrm{cms}^{-1}\right)\end{array}$ & $\begin{array}{c}\left|W_{1}\right| \\
\left(10^{-4} \mathrm{eV}\right)\end{array}$ & $\begin{array}{c}\xi_{0} \\
\left(10^{-5} \mathrm{~cm}\right)\end{array}$ & $\begin{array}{c}\lambda_{L 0} \\
\left(10^{-6} \mathrm{~cm}\right)\end{array}$ \\
\hline 2 & 1.203 & 1.30 & 1.29 & 20 & 0.1667 & 148.8 & 3.279 & 1.71 & 4.73 & 2.81 & 1.84 & 3.26 & 7.36 \\
\hline 3 & 1.203 & 1.56 & 1.58 & 27 & 0.1667 & 148.7 & 2.967 & 2.31 & 4.28 & 3.44 & 1.84 & 3.98 & 6.03 \\
\hline 4 & 1.267 & 1.11 & 1.12 & 15 & 0.1681 & 141.6 & 3.609 & 1.28 & 5.47 & 2.32 & 1.93 & 2.98 & 8.92 \\
\hline 5 & 1.356 & 1.07 & 1.07 & 12.6 & 0.1701 & 132.0 & 3.825 & 1.07 & 6.21 & 2.07 & 2.07 & 2.39 & 10.0 \\
\hline 6 & 1.154 & 2.57 & 2.55 & 59 & 0.1655 & 155.3 & 2.287 & 5.04 & 3.36 & 5.79 & 1.82 & 6.70 & 3.58 \\
\hline
\end{tabular}

graph that the absolute experimental values of $j_{c}(t)$ are given fairly accurately by

$$
\left.\left.j_{c}(t)\right|_{\exp } \simeq j_{0}\right|_{\exp }\left(1-t^{2}\right)^{3 / 2},
$$

where $\left.j_{0}\right|_{\exp }=1.07 \times 10^{7} \mathrm{~A} / \mathrm{cm}^{2}$.

We recall that $j_{0}$ of Sample 5 in our approach was calculated via the following values of the associated parameters: $\theta=428 \mathrm{~K}, T_{c}=1.356 \mathrm{~K}, \lambda_{1}=0.1701, E_{F}=$ $12.6 \mathrm{k} \theta, y_{0}=132.0, \gamma_{0}=1.36 \mathrm{~mJ} / \mathrm{mol} \mathrm{K}^{-2}$, and $v_{g}=10 \mathrm{~cm}^{3} / \mathrm{gram}$-atom. In order now to calculate $j_{c}(t)$ for this sample, we need to take into account the $T$-dependence of all these parameters. It seems reasonable to assume that among them, $\theta, E_{F}$ and $v_{g}$ retain the values employed for them at $T=0$. This assumption enables us to calculate $y(t)$ for any " $t$ " via (13) (with $\lambda_{2}=0$ ); the resulting values are given in Table 2. We could now calculate $j_{c}(t)$ if we knew $\gamma(t)$, but about which we have no information. However, we know that the heat capacity of an SC has a marked non-linear dependence on $t$ as discussed, e.g. in general in [12] and for superconducting $\mathrm{Ga}$ in ([13], p. 411). We are hence led to calculate $\gamma(t)$ with the input of $j_{c}(t)$-rather than the other way around-via the following equation

$$
\left.j_{c}(t)\right|_{\exp }=\left.j_{c}\left(\theta, y(t), \gamma(t), v_{g}, E_{F}\right)\right|_{\mathrm{Th}},
$$

the LHS of which for any " $t$ " is taken to be given by (17) and the RHS is calculated via (11) with the input of $y(t)$ obtained by solving (13). These values of $\gamma(T)$ are included in Table 2. Considered together with the values of $y(t)$, they will be shown below to provide a microscopic justification of Bardeen's phenomenological Equation (1). It is also remarkable that $y(t)$ and $\gamma(t)$ enable one to obtain quantitative estimates of several other $t$-dependent superconducting parameters, viz., $s(t)=m^{\star}(t) / m_{e}, n_{s}(t)=N_{s}(t) / N_{s 0}, V_{F}(t)=V_{F}(t) / V_{F 0}, V_{c}(t)=V_{c}(t) / V_{0}, \xi_{T}(t)=$ $\xi(t) / \xi_{0}$, and $\lambda_{L r}(t)=\lambda_{L}(t) / \lambda_{L 0}$. The plots of these are discussed below.

\section{Discussion}

1) The $T$-dependence of $\gamma(T)$ in the context of $j_{c}(t)$ is a new feature of the approach followed here. We recall that, as is well known, $\gamma$ is usually defined via the equation 
Table 2. Obtained as detailed in Section 3.2, values of $y(t)$ and $\gamma(t)$ corresponding to the experimental values of $j_{c}(t)$ for Sample 5 in [1] for $0 \leq t \leq 1$.

\begin{tabular}{cccc}
\hline$t$ & $\left.j_{c}(t)\right|_{\text {exp }} \times 10^{6}\left(\mathrm{~A} / \mathrm{cm}^{2}\right)$ & $y(t)$ & $\gamma(t) \times 10^{-4}\left(\mathrm{~J} / \mathrm{mol} \cdot \mathrm{K}^{-2}\right)$ \\
\hline 0 & 10.7 & 132.0 & 13.6 \\
0.1 & 10.5 & 132.4 & 13.3 \\
0.2 & 10.1 & 133.6 & 12.6 \\
0.3 & 9.29 & 133.9 & 11.5 \\
0.4 & 8.24 & 139.9 & 10.0 \\
0.5 & 6.95 & 145.9 & 8.25 \\
0.6 & 5.48 & 155.9 & 6.38 \\
0.7 & 3.90 & 172.6 & 4.46 \\
0.8 & 2.31 & 203.2 & 2.60 \\
0.9 & 0.89 & 276.8 & 0.99 \\
0.95 & 0.33 & 384.5 & 0.36 \\
1 & 0 & - & - \\
\hline
\end{tabular}

$$
C_{p}=C_{v}=\gamma T+464.6(T / \theta)^{3},
$$

where $C_{p}\left(C_{V}\right)$ is the heat capacity of the SC at constant pressure (volume) at very low temperatures $(<10 \mathrm{~K})$. The experimental data are usually plotted in the form $C_{v} / T$ vs. $T^{2}$, which yields an intercept equal to $\gamma$ and a slope equal to $464.6 / \theta^{\beta}$. The generally reported values of $\gamma$ in the literature, e.g. in [11], obtained in this manner correspond to $T=0$. It should also be noted that the simple relation (19) is invalid when the magnetic and nuclear contributions may be significant and, importantly, that $\gamma$ is directly proportional to $N\left(E_{F}\right)$, the density of states of electrons at the Fermi level. The latter of these features implies that we are taking into account the $T$-dependence of $N\left(E_{F}\right)$ via $\gamma(T)$.

2) It was assumed above that among the five parameters that are required for the calculation of $j_{c}(t)$ via (11), we need to take into account the $T$-dependence of only two of them, viz., $y$ and $\gamma$. Since the $T$-dependence of $j_{c}(t)$ is then governed by $R(t) \equiv[\gamma(t)]^{2 / 3} / y(t)$, we give for Sample 5 in [1] a plot of $R(t)$ vs. $t$ in Figure 1 , where $\gamma(t)$ and $y(t)$ are obtained via solutions of (18) and (13), respectively. Included in this figure is a plot of the ratio $R_{1}(t)=\left[\gamma(0)^{2 / 3} / y(0)\right]\left(1-t^{2}\right)^{3 / 2}$ vs. $t$, which is seen to be almost indistinguishable from the plot of $R(t)$. It therefore follows that our approach based on the microscopic BSE provides a detailed theoretical justification of the phenomenological Bardeen Equation (1) for $j_{c}(t)$.

3) In the approach followed in [1], while both $j_{c}(0)$ and $j_{c}(t \neq 0)$ depend on $e, T_{c}$ $V_{F}, \rho_{F}$ and $\ell$, the expression for the latter requires additional parameters as is seen from the Appendix in [1]. In the approach followed in this paper, no such additional parameters are required to deal with $j_{c}(t \neq 0)$; one simply invokes, where applicable, the $T$-dependence of the parameters on which $j_{c}(0)$ depends, viz. $e, \theta, y_{0}, \gamma_{0}, v_{g}$ and $E_{F}$. The two approaches may therefore be said to complement each other. 


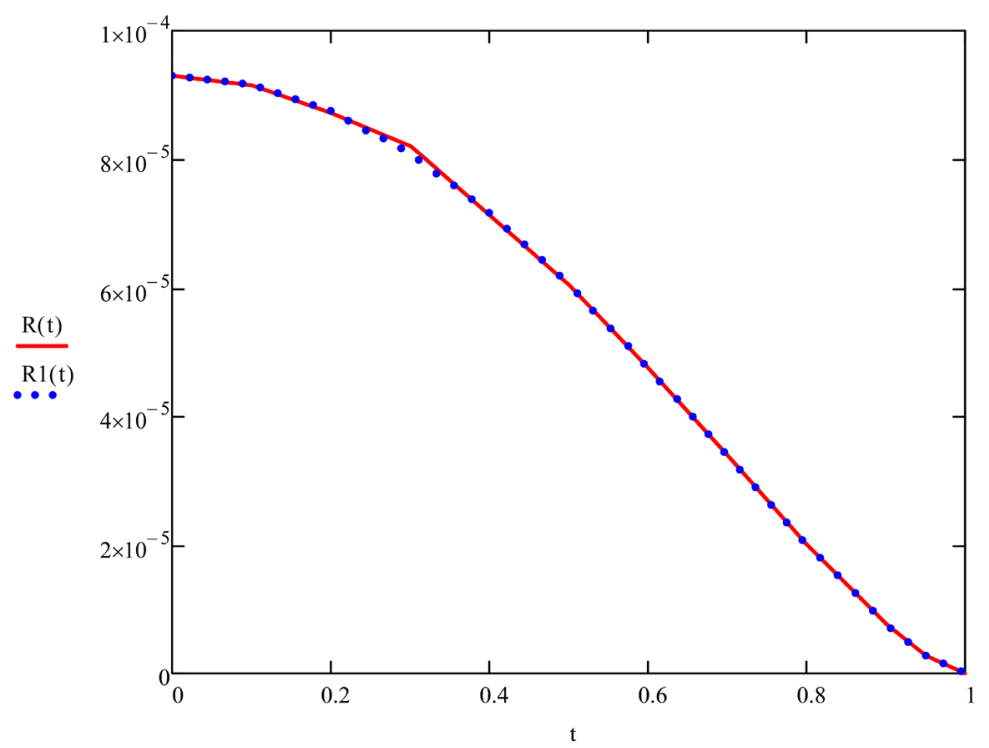

Figure 1. For Sample 5 in [1]: plots of $R(t) \equiv[\gamma(t)]^{2 / 3} / y(t)$ as obtained via solutions of (18) and (13), respectively, and of $R_{1}(t)=\left[\gamma(0)^{2 / 3} / y(0)\right]\left(1-t^{2}\right)^{3 / 2}$.

4) The expression for $j_{c}(0)$ in [1] depends on $V_{F}$, which is not so in the GBCSEs-based approach where $j_{c}(0)$ depends on $V_{c}(0)$. Nonetheless, it is interesting to note that the value of $V_{F}$ in [1] is assumed to be $1.36 \times 10^{8} \mathrm{~cm} / \mathrm{s}$ for all samples and is not $T$-dependent whereas, in the approach followed here, it differs from sample to sample and is $T$-dependent. For Sample 5, it varies between $(2.07-6.95) \times 10^{7} \mathrm{~cm} / \mathrm{sec}$ for $0 \leq t \leq 1$. For this sample, the values of $\xi_{0}$ and $\lambda_{L 0}$ too differ in the two approaches: while reported in [1] for these parameters are, respectively, the values $1.32 \times 10^{-4} \mathrm{~cm}$ and $1.10 \times 10^{-4} \mathrm{~cm}$, the corresponding values determined by us are $2.39 \times 10^{-5} \mathrm{~cm}$ and $1.00 \times 10^{-5} \mathrm{~cm}$.

5) Given in Figure 2 are the plots of $w_{1}(t), s(t)$ and $n_{s}(t)$ vs. $t$ for Sample 5 in [1]. Among these, even though the plot of $w_{1}(t)$ is obtained via an $E_{F}$-incorporated GBCSE with $E_{F}=12.6 \mathrm{k} \theta$, it is very similar to the plot one obtains for $\Delta(T) / \Delta_{0}$ for an elemental SC via the usual BCS equation sans $E_{F}$. While we could not find any experimental data for the parameter $s(t) \equiv m^{*}(T) / m_{e}$ for the SC under consideration, we draw attention to a plot of this parameter for $\mathrm{Pb}$ and Ta given in (Figure 7 of [12]). This plot covers temperatures up to about $120 \mathrm{~K}$ and therefore does not specifically shed light on the behavior of $s(t)$ in the superconducting state. Nonetheless, it is notable that it displays a parabolic decrease for a major part of the range of temperatures over which it is plotted. As for $n_{s}(t)$, as shown in Figure 2, we find that a good analytic fit to the values calculated by us is provided by $n_{s}(t)=\left(1-t^{2}\right)^{1.51}$, which is at variance with the result of the simple two-fluid model where $n_{s}(t)=\left(1-t^{4}\right)$. However, it is also well known that, factually, $T$-dependences of superconducting parameters often differ from those following from the simple two-fluid model ([11], p. 48).

6) In the context of Figure 3 which is the plot of the reduced fermi velocity $v_{F}$ that our approach has led to, we draw attention to a paper by Plumb et al. [14] 
who have reported that "Associated with this feature (a kink-like feature observed at extremely low energy along the superconducting node in Bi-2212), the Fermi velocity scales substantially-increasing by roughly $30 \%$ from 70 to $110 \mathrm{~K}$. The temperature dependence of the feature suggests a possible role in superconductivity, although it is unclear at this time what mechanism(s) may lead to this low-energy renormalization". We are hence led to suggest that Figure 3 provides both: a plausible explanation that Plumb et al. sought for their result and a validation of our approach.

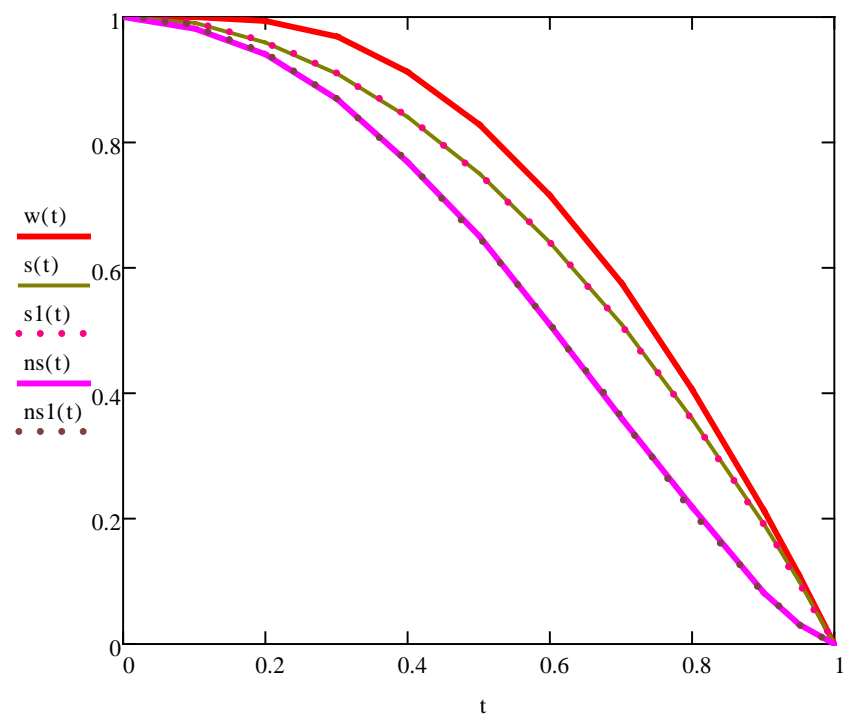

Figure 2. For Sample 5 in [1]: clockwise, solid lines represent $w(t)=W_{1}(T) / W_{10}$, $s(t)=m^{*}(T) / m_{e}$, and $n_{s}(t)=N_{s}(T) / N_{s 0}$ calculated via, respectively, (3), and the T-generalized (7) and (8). For values of $W_{10}, s(0)$ and $N_{s 0}$, see Table 1 . The dotted plot overlapping $s(t)$ is the analytic fit to it obtained via $s_{1}(t)=\left(1-t^{2}\right)$; the plot overlapping $n_{s}(t)$ is a similar plot obtained via $n_{s 1}(t)=\left(1-t^{2}\right)^{1.51}$.

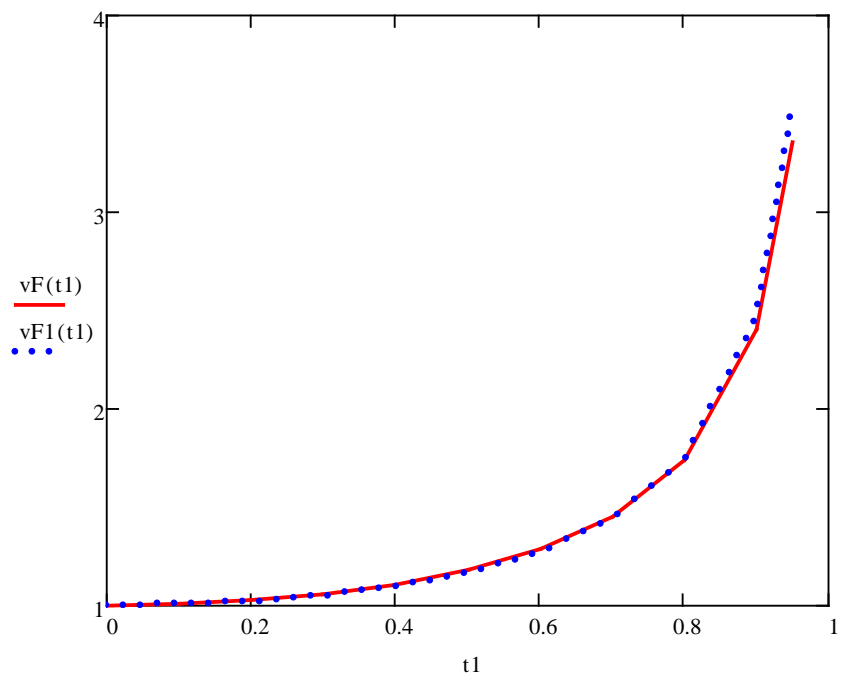

Figure 3. Plot of the reduced fermi velocity $v_{F}(t)=V_{F}(T) / V_{F 0}$ corresponding to Sample 5 in [1]. The dotted plot overlapping it is obtained via $v_{F 1}\left(t_{1}\right)=\left(1-t_{1}^{2}\right)^{-0.54}$. 
7) Both $\xi_{r}(t)$ and $\lambda_{L r}(t)$ are known to diverge at $t=1$, which is a feature also reflected in our Figure 4 and Figure 5 . It is thereby seen that for the former parameter, $\xi_{1}(t)=\left(1-t_{1}^{2}\right)^{-1.41}$ provides a good fit to $\xi_{r}(t)$, whereas a similar fit for the latter parameter is provided by $\lambda_{L 1}(t)=\left(1-t^{2}\right)^{-0.27}$.

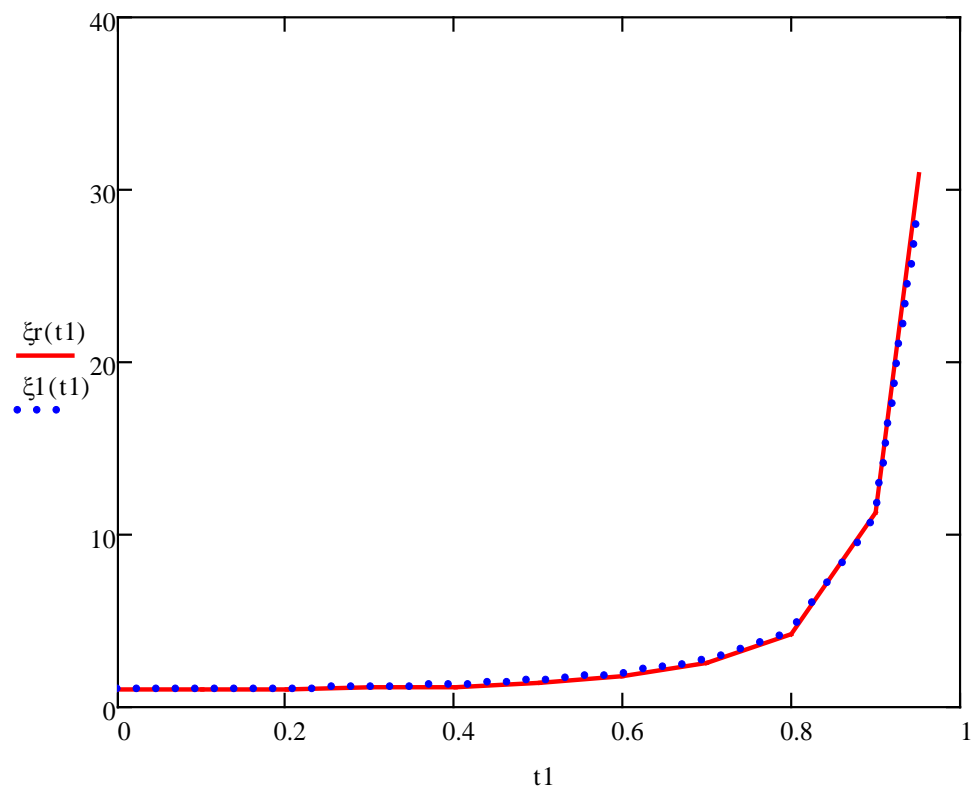

Figure 4. For Sample 5 in [1], the solid line is the plot of the reduced coherence length $\xi_{r}(t)=\xi(T) / \xi_{0}$ as obtained via theory in this paper. The overlapping plot is obtained via $\xi_{1}\left(t_{1}\right)=\left(1-t_{1}^{2}\right)^{-1.41}$.

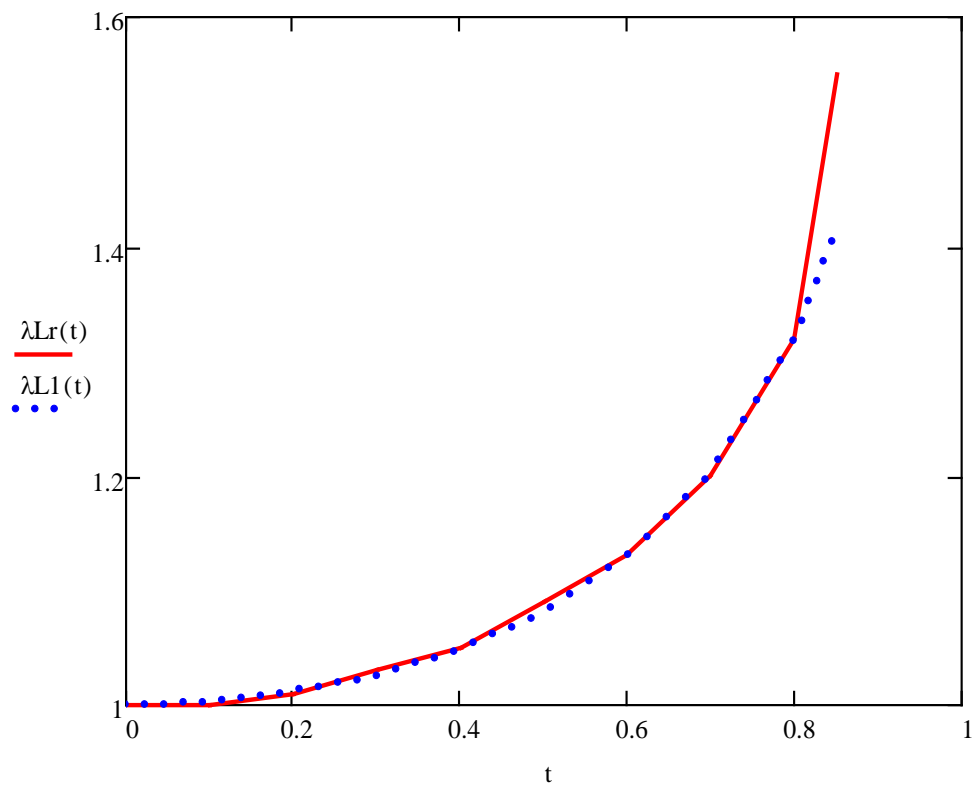

Figure 5. For Sample 5 in [1], the solid line is the plot of the reduced London penetration depth $\lambda_{\mathrm{Lr}}(t)=\lambda_{L}(t) / \lambda_{L 0}$ as obtained via theory in this paper. The overlapping plot is obtained via $\lambda_{L 1}(t)=\left(1-t^{2}\right)^{-0.27}$. 
8) As concerns the rather accurate numerical fits that we have obtained for the values of various empirical parameters associated with $j_{\mathcal{c}}$, it is remarkable that each of them is found to vary as some power of $\left(1-t^{2}\right)$. Viewed in conjunction with (1), it provides another example of the deep physical insight that Bardeen had without the benefit of a detailed microscopic theory governing $j_{c}$.

\section{Conclusions}

It has been shown above that the GBCSEs-based approach provides a valuable alternative to the explanation of the Romijn et al's $j_{c}(T)$ empirical data for superconducting Al strips based on the KL [3] approach derived from the Eilenberger equations which, in turn, follow from the microscopic Gor'kov theory when certain simplifying assumptions are made. Unique features of the GBCSEs-based approach are: 1$)$ by appealing to the $j_{c}(0)$ value of an SC, it leads to a unique value of $E_{F}$ that enables one to deal with its $\left\{\Delta, T_{c}, j_{c}(T)\right\}$ values in a unified framework, 2) with $E_{F}$ thus fixed, appeal to the $j_{c}(t)$ values of the SC leads to a new finding about how $\gamma(t)$ varies with $t$, which is then shown to lead to (3 quantitative estimates of several $T$ - and $E_{F}$-dependent superconducting parameters, viz., $s(t)$, $n_{s}(t), V_{c}(t), V_{F}(t), \xi_{r}(t)$ and $\lambda_{L r}(t)$. It is remarkable that one can obtain these results by remaining within the ambit of the mean-field approximation, i.e. by employing the model (constant) BCS interaction " $-V$ ", which for (6) in the scenario of $1 \mathrm{PEM}$ is operative only when $E_{F}-k \theta \leq(\boldsymbol{P} / 2+\boldsymbol{p})^{2} / 2 m$, $(\boldsymbol{P} / 2-\boldsymbol{p})^{2} / 2 m \leq E_{F}+k \theta$ and vanishes otherwise ([7], p. 117); for (2), (3) and (4), the corresponding constraints on $\mathrm{V}$ are obtained by putting $\boldsymbol{P}=0$ in these inequalities and are identical with those in the usual BCS theory.

As was mentioned above, a plethora of formulae is known in the literature for calculating $j_{c}$ of an SC, depending upon its type (I or II), size, shape and the manner of preparation. The application of $E_{F}$-incorporated GBCSEs herein, and to a variety of other SCs in [8] (with a correction in [10]), suggests that the $E_{F}$ of an SC subsumes most of these properties.

We conclude by noting that work is in progress to further generalize the GBCSEs given here to deal with the pragmatic situation where the SC is in a heat bath in an external magnetic field, i.e. when both $T$ and $H$ are non-zero-a procedure for which has been given in [15].

\section{Conflicts of Interest}

The authors declare no conflicts of interest regarding the publication of this paper.

\section{References}

[1] Romijn, J., Klapwijk, T.M., Renne, M.J. and Mooij, J.E. (1982) Critical Pair-Breaking Current in Superconducting Aluminum Strips far below $T_{c}$. Physical Review B, 26, 3648-3655. https://doi.org/10.1103/PhysRevB.26.3648

[2] Bardeen, J. (1962) Critical Fields and Currents in Superconductors. Reviews of 
Modern Physics, 34, 667-681. https://doi.org/10.1103/RevModPhys.34.667

[3] Kupriyanov, M.Y. and Lukichev, V.F. (1980) Temperature Dependence of Pair-Breaking Current in Superconductors. Soviet Journal of Low Temperature Physics, 6, 210.

[4] Suhl, H., Matthias, B.T. and Walker, L.R. (1959) Bardeen-Cooper-Schrieffer Theory in the Case of Overlapping Bands. Physical Review Letters, 3, 552-554. https://doi.org/10.1103/PhysRevLett.3.552

[5] McMillan, W.L. (1968) Transition Temperature of Strong-Coupled Superconductors. Physical Review, 167, 331-344. https://doi.org/10.1103/PhysRev.167.331

[6] Malik, G.P. (2013) On a New Equation for Critical Current Density Directly in Terms of the BCS Interaction Parameter, Debye Temperature and the Fermi Energy of the Superconductor. World Journal of Condensed Matter Physics, 3, 103-110. https://doi.org/10.4236/wjcmp.2013.32017

[7] Malik, G.P. (2016) Superconductivity: A New Approach Based on the Bethe-Salpeter Equation in the Mean-Field Approximation (Series on Directions in Condensed Matter Physics Book 21). World Scientific, Singapore. https://doi.org/10.1142/9868

[8] Malik, G.P. (2016) On the Role of Fermi Energy in Determining Properties of Superconductors: A Detailed Comparative Study of Two Elemental Superconductors $(\mathrm{Sn}$ and $\mathrm{Pb})$, a Non-Cuprate $\left(\mathrm{MgB}_{2}\right)$ and Three Cuprates (YBCO, Bi-2212 and Tl-2212). Journal of Superconductivity and Novel Magnetism, 29, 2755-2764. https://doi.org/10.1007/s10948-016-3637-5

[9] Malik, G.P. (2017) A Detailed Study of the Role of Fermi Energy in Determining Properties of Superconducting NbN. Journal of Modern Physics, 8, 99-109. https://doi.org/10.4236/jmp.2017.81009

[10] Malik, G.P. (2018) Correction to: On the Role of Fermi Energy in Determining Properties of Superconductors: A Detailed Comparative Study of Two Elemental Superconductors ( $\mathrm{Sn}$ and $\mathrm{Pb}$ ), a Non-Cuprate $\left(\mathrm{MgB}_{2}\right)$ and Three Cuprates (YBCO, Bi-2212 and Tl-2212). Journal of Superconductivity and Novel Magnetism, 31, 941-941. https://doi.org/10.1007/s10948-017-4520-8

[11] Poole, C.P. (2000) Handbook of Superconductivity. Academic Press, San Diego.

[12] Kresin, V.Z. and Zaĭtsev, G.O. (1978) Temperature Dependence of the Electron Specific Heat and Effective Mass. Soviet Physics JETP, 47, 983.

[13] Kittel, C. (1974) Introduction to Solid State Physics. Wiley Eastern, New Delhi.

[14] Plumb, N.C., et al. (2009) Low-Energy (< $10 \mathrm{meV})$ Feature in the Nodal Electron Self-Energy and Strong Temperature Dependence of the Fermi Velocity in $\mathrm{Bi}_{2} \mathrm{Sr}_{2} \mathrm{CaCu}_{2} \mathrm{O}_{8+\delta}$ https://arxiv.org/pdf/0903.4900.pdf

[15] Malik, G.P. (2010) On Landau Quantization of Cooper Pairs in a Heat Bath. Physica $B, 405,3475-3481$. https://doi.org/10.1016/j.physb.2010.05.026 